\title{
Self-stimulation in pigeons: Tests of two alternative explanations'
}

EUAN M. MACPHAIL, LABORATORY OF EXPERIMENTAL PSYCHOLOGY, THE UNIVERSITY OF SUSSEX, Falmer Brighton, Sussex, England

Intracranial stimulation in pigeons has previously been shown to be capable of sustaining extinction responding in hungry birds (Macphail. 1967). Experiment 1 was designed to test the hypothesis that this effect was due to the disruption by brain-shocks of the extinction process; it was found that brain-shocks had no effect on a task involving a series of reversals. The notion that brain-shocks have the effect of prolonging extinction through having acquired secondary reward value was shown to be untenable in Experiment 2. It was concluded that the brain-shocks have a positively reinforcing effect comparable to that responsible for selfstimulation in mammals.

In previous experiments it has been found that electrical stimulation of the brain (ESB) delivered to hungry pigeons for responses during extinction following food-rewarded sessions may maintain extinction responding (Macphail, 1967), but that most birds showing this effect do not work for ESB in the absence of prior food-rewarded sessions, despite the delivery of "priming" shocks (Macphail, 1966). The two experiments reported here were designed to test the plausibility of two possible explanations of the effects found, alternative to the view that the ESB is acting as a primary reinforcer.

\section{EXPERIMENT 1}

Experiment 1 tests the possibility that the effect of ESB is in fact not to positively reinforce behavior, but rather to disrupt the extinction process.

Method. Subjects were five adult pigeons (Columba livia) each having an implanted pair of bipolar electrodes in the forebrain; all had been shown to respond reliably during extinction periods where each response obtained an $0.4 \mathrm{sec}$ train of ESB. The Ss were maintained at $80 \%$ ad lib weight throughout the experiment.

The apparatus was a modified Skinner box, with two keys. In pretraining each key was exposed singly, and Ss learned to respond at FR10 for food reward.

In test sessions, both keys were exposed, and the first response, to whichever key, was rewarded; three food rewards were then delivered at FR10 on that key, responses to the other key being scored as errors and having no effect on rewards. After these three rewards, the reinforcement value of the keys was reversed, responses on the original key now counting as errors, and food being delivered at FR10 on the other key. The reinforcement value of the keys was reversed in this way, every 30 "correct" responses, until the bird had obtained 25 food rewards (i.e., had made 240 correct responses).

In control sessions, no ESB was delivered at any stage, whereas in experimental sessions, an $0.4 \mathrm{sec}$ train of ESB was delivered for every response, to whichever key. The intensity of ESB was set for each $S$ at a level previously shown to maintain extinction responding in that $\mathbf{S}$. There were two sessions per day, and five days testing. The first two sessions were control sessions, run to ensure reliable responding by the $S s$, and error scores on those sessions were discarded. On the next four days, one session was experimental, and one, control; the first session of the first and third of these days was a control session, and on the second and fourth days, experimental.

Results and Discussion. There were no significant differences between the error scores of the experimental and control sessions, and there was considerable overlap of scores for the two conditions in all five cases.

Analysis of errors showed that the birds responded systematically in this situation, making more errors following a reversal of the correct key than after obtaining the first of the series of three rewards from a correct key. There is, therefore, reason to suppose that, although reversal and extinction are not identical situations, had these birds been incapable of extinction, their performance in this task would have been severely impaired; evidence from studies of mammalian brain function shows that, when extinction is impaired by a lesion, reversal is also impaired (Douglas, 1967; Gerbrandt, 1964). In the light of this result, the notion that the reward effect in pigeons is an an artifact of the disruption of the extinction process by ESB is now implausible.

\section{EXPERIMENT 2}

A second alternative account of the significance of the acquisition period in obtaining the reward effect in pigeons is the notion that ESB is rewarding, but has become so only in virtue of its association with food reward during the acquisition periods; that is, the phenomenon is one of secondary reward.

Experiment 2 was designed to answer two questions relevant to this notion; first, will the "rewarding" ESB become aversive as a consequence of being explicitly paired with extinction, and second, is "neutral" ESB capable of acting efficiently as a signal for the beginning of extinction?

Method. The Ss were nine adult pigeons (Columba livia) each having an implanted pair of bipolar electrodes in the forebrain. ESB delivered to the Reward Group ( $N=4)$ had been shown to prolong extinction responding, whereas ESB to the Neutral Group $(N=5)$ had been shown not to affect extinction performance. Both groups were maintained at $80 \%$ ad lib weight throughout the experiment.

The Ss were tested in a single key Skinner box. There were four sessions each day; each session consisted of an acquisition period, in which 10 food rewards were delivered at FR10, and a 5-min extinction period. In control sessions, no ESB was delivered; in experimental sessions an $0.4 \mathrm{sec}$ train of ESB was delivered for each response in extinction periods. The intensity of ESB was $100 \mu \mathrm{A}$ for the Neutral Group, and at a level shown to sustain extinction period responding for each individual in the Reward Group.

The Ss were tested on five consecutive days. All sessions on the first four days were experimental; on the fifth day, the first and third sessions were control, the second and fourth being experimental.

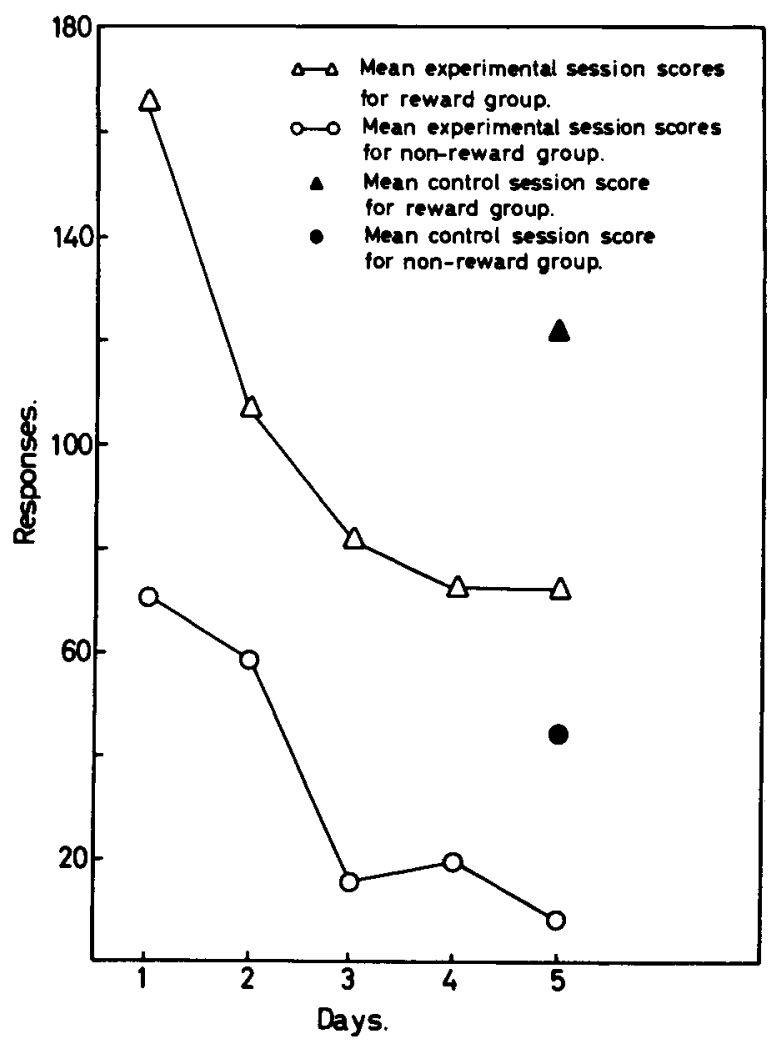

Fig. 1. 
Results and Discussion. A graph showing the mean extinciton period scores for the two groups is presented in Fig. 1. The mean scores for each day of the experiment are plotted, and show a progressive fall in responding in both groups.

Examination of individual scores reveals that, for both groups, the scores of the experimental sessions on the fifth day are reliably lower than those of the control sessions, there being no overlap in any $\mathrm{S}$.

The difference between the experimental scores of the two groups on the fifth day was significant, as was the difference between their control scores for that day (both at better than the .01 level, by the $t$ test).

This experiment was designed so that the beginning of each extinction period in experimental sessions coincided with the first delivery of ESB. Two of the results obtained contradict what might have been expected on the secondary reward hypothesis; first, ESB acts extremely efficiently for the Neutral Group as a signal that extinction has commenced, so that there is no plausible reason why the ESB delivered to this group should not also have acquired secondary reinforcing properties in the original experiment. Second, after five days testing there was still a large difference between the extinction scores of the two groups, and reason to believe, from the flattening of the Reward Group curve, that this difference would persist. That is, despite the explicit association of ESB with nonreward, ESB maintains its ability to reinforce behavior.

The results of the two experiments reported here support the view that the phenomenon found in pigeons (Macphail, 1967) is a genuine instance of ESB acting as a primary reinforcer, although it is clear that more research is needed before a satisfactory account of its peculiarities (Macphail, 1966) can be attempted.

\section{REFERENCES}

DOUGLAS, R. J. The hippocampus and behavior. Psychol. Bull., 1967, 67, $416-442$.

GERBRANDT, L. K. Neural systems of response release and control. Psychol. Bull, 1965, 64, 113-123.

MACPHAIL, E. M. Self-stimulation in pigeons: The problem of "priming." Psychon. Sci., 1966, 5, 7-8.

MACPHAIL, E. M. Positive and negative reinforcement from intracranial stimulation in pigeons. Nature, 1967, 213, 947-948.

\section{NOTE}

1. The research reported in this document has been sponsored by the Air Force Office of Scientific Research under Grant AF EOAR 642 through the European Office of Aerospace Research (OAR), United States Air Force, and was carried out at the Institute of Experimental Psychology, Oxford University, while the author held a UK Medical Research Council Scholarship for training in Research Methods. 